\title{
Optics in India: The Spectroscopic Behavior of Rock Salt
}

\author{
C. V. Raman
}

\begin{abstract}
The origin of the infrared activity of rock salt is discussed in terms of the free vibrations of the crys tal. Experimental data on the Reststrahlen frequencies and on the frequency displacements of scattered radiation are used to explain the spectroscopic behavior of rock salt in the near infrared.
\end{abstract}

Rock salt was one of the first minerals of which the crystal structure was established by x-ray analysis. Its structure may be described in simple terms as made up of two interpenetrating lattices, each composed of atoms of one kind. The sodium atoms in one lattice are surrounded by six chlorine atoms in the other lattice and vice versa, in such manner that the grouping exhibits cubic symmetry. The sodium and chlorine atoms appear interspersed with each other in the cubic planes of the structure, while in the octahedral planes they appear separately in alternate layers.

The transparency of rock salt over a wide spectral range extending from $0.2 \mu$ to $10 \mu$ makes it a material of first-rate importance in applied optics and especially for use as dispersing prisms in the infrared. The dispersive power of rock salt is very low, between $1 \mu$ and $5 \mu$, but improves at greater wavlengths. On the other hand, the absorption which is negligible at $10 \mu$ becomes sensible at $13 \mu$ and increases rapidly beyond $15 \mu$. Hence, rock salt may best be used as a prism material for infrared spectroscopy between $7 \mu$ and $14 \mu$. It ceases to be useful beyond $15 \mu$.

It is well known that rock salt exhibits, in the region of long wavelengths, a high reflecting power which reaches its maximum value of about $90 \%$ at $54 \mu$. The strength of this reflection falls off rapidly at shorter wavelengths and is negligibly small for all wavelengths less than $33 \mu$. The fall is less rapid for wavelengths greater than $54 \mu$ and the reflecting power reaches a limiting value of about $20 \%$ at $100 \mu$. These facts call for explanation; in particular, it may be

The author is the director of the Raman Research Institute,

Bangalore, India.

Received 2 February 1962. asked why, at $15 \mu$ which is outside the spectral region of strong reflections, the absorbing power is nevertheless large enough to impair the usefulness of the material for infrared spectroscopy.

It is proposed in this paper to review briefly the contents of a memoir by the author which has been recently published and in which the spectroscopic behavior of rock salt has been discussed in detail. ${ }^{1}$

\section{The Free Vibrations of the Structure}

The dispersion, absorption, and reflection of infrared radiation by a crystal are effects arising from the interaction of the electromagnetic field in the radiation with the structural units composing the crystal. As a first step toward an understanding of these effects, it is necessary to consider the nature of the spectrum of the free or spontaneous vibrations of these structural units. We may deduce their modes and frequencies by the methods of classical mechanics. For this purpose, the atomic nuclei may be regarded as simple mass particles and the electronic clouds surrounding them as massless springs which hold the structure together. The relevant theory is fully set out in the memoir cited above and it is sufficient here to state the results to which it leads.

It emerges that the vibrational mode of highest frequency of the rock salt structure is one in which the atoms of sodium and chlorine oscillate against each other in opposite phases. Eight other modes of free vibration are also possible. They may be described simply in terms of the crystal structure: four of the modes are oscillations of the cubic layers, while the other four modes are oscillations of the octahedral layers, the movement alternating in phase from layer to layer, and being either normal or tangential to those layers. Since the oscillations of the sodium and 
chlorine atoms may be either in the same phase or in opposite phases, we have four modes for the cubic layers and four modes for the octahedral layers, and hence eight in all. The oscillations of the sodium and chlorine atoms located in the same cubic layers would be coupled with each other. But the oscillations of the sodium and chlorine atoms, appearing as distinct layers in the octahedral planes, would be independent.

\section{The Origin of Infrared Activity}

We next proceed to consider the relation between the free vibrations of the structure of the crystal and the effects arising from the passage of infrared radiation through it. The electric field in the incident radiation acts both on the positively charged nuclei and on the negatively charged electrons. But in all the modes of vibration with which we are concerned, there are as many positively charged nuclei moving in one phase as there are others moving in the opposite phase. It can be shown that, as a consequence, the displacements of positive charge when totaled up cancel out in all the modes under consideration. Hence, the free vibrations of the structure cannot be excited by the forces exerted by the field on the atomic nuclei. Necessarily, therefore, the movements of the atomic nuclei can have no effect on the radiation passing through the crystal. We conclude that the infrared activity of the crystal arises solely by reason of the forces exerted by the field on the negatively charged electrons and of the resulting movements; such activity is only possible when the displacements of negative charge produced do not cancel out when summed up over each structural unit.

Since the electrons are held in position by their interactions with each other and with the positivelycharged nuclei, the displacements of negative charge induced by the field would necessarily disturb the nuclei from their positions of equilibrium. But the nuclear movements thus arising would not contribute to the infrared activity. Thus, while in the free vibrations of the structure, the massive, positively charged nuclei play the leading role and the electrons a subsidiary one, the situation is reversed in respect of the infrared activity, the electrons here playing the leading role and the nuclei a subsidiary one. In other words, the spectrum of free vibrations and the spectrum of infrared absorption are essentially different in their nature and origin. Both being properties of the same structure, we may expect to find some resemblances, but nothing in the nature of an identity or complete correspondence is to be expected.

\section{The Reststrahlen Reflections}

The considerations set forth above indicate that the mode of vibration in which the sodium and the chlorine atoms oscillate in opposite phases would be that exhibiting by far the strongest infrared activity. Their approach and recession would be accompanied by a periodic movement of the electronic cloud located between each pair of nuclei. The effects due to the displacements of electric charge in the successive cells of the structure would be additive and would thereby attain a large magnitude. Indeed, the activity may be expected to be so great that secondary radiations would emerge from the units of the structure along the external boundary of the crystal and conspire to give a powerful surface reflection. There would also be a strong absorption and extinction of the radiation actually penetrating into the crystal.

As a matter of actual observation, the surface reflection appears with great strength between $50 \mu$ and $60 \mu$, its maximum of intensity being at $54 \mu$. On the other hand, the minimum of transmission by very thin films of rock salt appears at about $60 \mu$, being thus displaced a little further into the infrared. In what follows, we shall take $56 \mu$ to be the wavelength corresponding to the frequency of the strongly active mode of free vibration of the rock salt structure.

\section{Infrared Activity of Higher Orders}

The absorption of infrared radiation arises when the electromagnetic energy of the field is taken up and transformed into vibrational energy in the crystal. When the frequencies of the field and the mechanical vibration are identical, a quantum of radiational energy changes over to a quantum of vibrational energy. There are also other possibilities: the energy of the radiation taken up by the crystal may appear as two, three, or a larger number of quanta of vibrational energy. Thus, we are led to expect, besides the intense absorption around $56 \mu$, a series of other absorptions around $28 \mu, 19 \mu$, and $14 \mu$, respectively. They may be referred to as the absorptions of the second, third, and fourth orders, respectively. The possibility of observing the higher orders of absorption arises by reason of the first-order absorption being itself of great strength. The absorptions of higher order would necessarily be weaker and of progressively diminishing strength with increasing order. Nevertheless, when the absorption path is sufficiently increased, their effect on the transmission should be observable.

That the lack of transparency of rock salt at wavelengths greater than $15 \mu$, which renders it unusable as material for dispersing prisms, is due to these absorptions of higher order may be inferred from the observed facts of the case. The absorption at $14 \mu$ is noticeable when the thickness of the plate is $5 \mathrm{~cm}$ or more, but it ceases to be significant when the absorption path is reduced to $3 \mathrm{~cm}$ or less. Yet in this range of thickness, there is a complete cutoff of the incident 
radiation commencing at $19 \mu$ and extending to greater wavelengths. Reducing the thickness to $15 \mathrm{~mm}$ produces but little observable change in this behavior, the wavelength of cutoff drifting slightly to $20 \mu$. Not until the thickness is reduced to $1 \mathrm{~mm}$ or so does the transmission at $19 \mu$ become at all comparable with that observed at $14 \mu$ with far larger thicknesses. To obtain such a free transmission at $28 \mu$, the path has to be further reduced to about $50 \mu$, while only films which are a few microns thick would give an observable transmission at $56 \mu$. It is evident from such facts that we are dealing with absorptions of altogether different orders of magnitude in these four cases.

\section{The Infrared Activity of the Other Modes}

We have now to consider the eight other modes of free vibration mentioned earlier. By reason of the vibrations being in opposite phases in the alternate layers, the displacements of electric charge in all the eight modes cancel out in the first approximation. But in the case of the four coupled oscillations of the sodium and chlorine atoms appearing in the cubic layers, the displacements of electric charge do not cancel out in the second approximation; there is a surviving component having twice the frequency of the mode under consideration. Hence these coupled vibrations would be strongly infrared active, but with doubled frequency in each case.

Taking $56 \mu$ as the wavelength of maximum infrared activity, the wavelengths corresponding to the four modes referred to may be theoretically estimated to be $66 \mu, 78 \mu, 90 \mu$, and $108 \mu$, respectively. The halved wavelengths at which their second-order activity would manifest itself would therefore be $33 \mu$, $39 \mu, 45 \mu$, and $54 \mu$, respectively.

The foregoing figures enable us to understand why the spectral region in which the strong surface reflections appear extends toward shorter wavelengths up to $33 \mu$ and terminates at that wavelength. This extension is clearly the result of the superposition of the effects due to these modes and that of the mode of greatest activity. The activities of these modes appearing at $33 \mu, 39 \mu, 45 \mu$, and $54 \mu$ are likewise responsible for the features noticeable in the spectral transmission curves of thin plates and thin films of rock salt at these wavelengths. These features have been observed and reported by Mentzel and by Barnes and Czerny in their studies on the infrared behavior of rock salt.

By reason of their symmetry properties, the vibrations of the sodium and chlorine atoms appearing in the layers parallel to the octahedral planes can exhibit no infrared activity. They, and indeed all the other modes as well, are accessible to observation by other spectroscopic methods. In this way, all the nine characteristic wavelengths of rock salt can be determined: viz., $56 \mu, 59 \mu, 64 \mu, 66 \mu, 71 \mu, 78 \mu, 85 \mu, 90 \mu$, and $108 \mu$. These wavelengths form a nearly continuous sequence extending into the region of great wavelengths far beyond that at which the maximum intensity of surface reflection appears. We are thereby enabled to understand why the spectral region, in which strong surface reflections appear, extends up to $100 \mu$. An intense excitation of the electrons in a thin layer adjacent to the surface of the crystal must of necessity involve all the nine possible modes of vibration and not only that of the mode of highest frequency. The strength of the excitation of any particular mode would be determined by the difference between its frequency and that of the most highly active mode. It would, therefore, fall off steadily as we recede from the mode having the highest activity towards lower frequencies and longer wavelengths.

\section{The Spectrum of Light Scattering}

The picture of the dynamical behavior of the rock salt structure and its relation to the infrared behavior of the crystal briefly summarized above finds a striking confirmation in the results of two other entirely independent lines of research. The first is the evaluation of the thermal energy of rock salt over the whole range of temperatures extending from absolute zero upwards, the theoretically determined and spectroscopically confirmed frequencies of vibration of the structure and their respective degeneracies being the basis of the calculation. To enter into this subject would take us beyond the field of applied optics; we shall therefore here only deal with the other line of investigation. This is a spectroscopic method closely related to the field of infrared studies, viz., the study of the spectral shifts observed in the scattering of monochromatic light in its passage through rock salt.

The frequency shifts observed in the scattering of light arise from the periodic changes in the optical scattering power of the structural units in the crystal. These changes are produced by their internal vibrations and hence the observed shifts may usually be identified with the frequencies of the vibrations. But in a particular class of cases, frequency shifts are recorded with notable intensity in the spectrum of the scattered light, which are double the frequency of the vibrations of the structure. The frequency shifts observed with rock salt are all of this kind, and the reason for this is not far to seek: In each of the nine modes of free vibration, we find a sodium atom approaching a chlorine atom on one side and simultaneously receding from a chlorine atom on the other side, and vice versa. The increased scattering power due to the approach is set off by the decrease due to the recession and cancels out in the first approximation. But in the second approximation, there is a residue left over, and this 


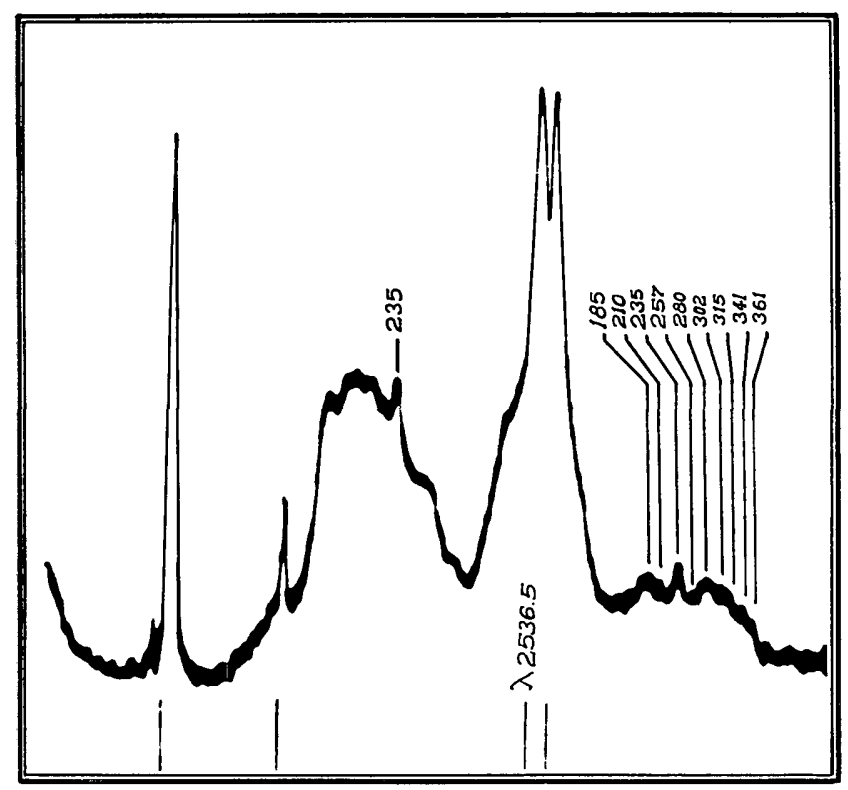

Fig. 1. Rasetti's microphotometer record of the rock salt spectrum.

would evidently be periodic but with a doubled frequency.

The spectrum of the scattering of light in rock salt was successfully recorded several years ago by Rasetti in a noteworthy investigation. The microphotometer record of the spectrum obtained by him is reproduced from his paper as Fig. 1. The frequency shifts in wavenumbers have been marked in the figure. The record shows the position of the exciting radiation $(2536.5 \AA)$ from a water-cooled mercury arc enclosed in quartz which was placed between the poles of an elec- tromagnet in order to eliminate the self-reversal of the emitted radiation. The frequency shifts were both negative and positive, and they appeared respectively to the left and to the right of the position of the exciting radiation in the microphotometer trace.

The negative shifts are recorded with great intensity and, being overexposed in the original spectrogram, are not so clearly resolved from each other in its microphotometer trace as the positive shifts. These latter appear as successive steps in the record, and the respective frequency shifts are easily read off from their displacements with respect to the $\lambda 2536.5$ line. It will be seen that all the nine different frequency shifts may be clearly recognized. Their values as read off from the record on the right-hand side are in excellent agreement with the doubled frequencies of the respective modes, as theoretically estimated.

The relative intensities of the nine negative-frequency shifts as indicated by Rasetti's microphotometer record are also in satisfactory accord with the estimates based on the nature of the individual modes and their respective degeneracies. It should be mentioned the frequency shifts of $\pm 235 \mathrm{~cm}^{-1}$ stand out in Rasetti's original spectrogram as sharply defined lines. They represent the doubled frequency of vibration of the chlorine atoms located in the octahedral layers in which the movements are parallel to these layers. The notable intensity of the $235 \mathrm{~cm}^{-1}$ line is a consequence of the eightfold degeneracy of the mode.

\section{Reference}

1. C. V. Raman, Proc. Indian Acad. Sci. Sec. A 54, 253-304 (1961).
1962

May

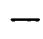

2

3

$6-10$

6-10

$7-11$

\section{Meetings Calendar}

$14-16$

$14-16$

$20-23$

21-25

IRE International Scientific Radio Union, Washington, D.C., Spring Meeting IRE, 1 E. 79th St.. New York 21, N.Y.

OSA President MacAdam's address to S. Calif. Section on Optical Engineering, Los Angeles, Calif.

OSA President MacAdam's address on Optical Engineering to Sigma Xi Club, San Diego, Calif.

French Society of Ophthalmology Congress, Paris Secrétaire Général Société Francaise d'Ophthalmologie, 27 rue du Faubourg-Saint-Jacques, Paris, France

Electrochemical Society Meeting, Statler Hilton Hotel, Los Angeles, Calif.

Society of Photographic Scientists and Engineers, Somerset Hotel, Boston, Mass. K. Kohlman, 13712 Mariana Dr., Rockville, $M d$.

OSA Rochester Section Annual Dinner Meeting J. L. Rood, Bausch \& Lomb, Inc., Rochester, N.Y.

Society for Applied Spectroscopy New York and Philadelphia Sections Solid State Lasers Meeting

23

$22-24$ 14th Annual National Aerospace Electronics Conf., Biltmore Hotel, Dayton, Ohio G. Langston, 4745 Rean Meadow Drive, Dayton 40, Ohio

Symp. on Thermionic Power Conversion, Colorado Springs V. C. Wilson, GE Company, Research Lab, Schenectady, N.Y.

Radiation Research Society 10th Annual Meeting, Colorado Springs

Max Planck Institute for the Advancement of Science, 13th General Assembly, Düsseldorf J. Ammundsen, Sammenslutningen of Arbejdsginere Indenfor den Keramiske Industri, Norrevolgade 34, Copenhagen, Denmark

OSA President MacAdam's address to First Meeting Wash. D.C. Section on Color in Court.

IRE National Microwave Theory and Techniques Symposium, Boulder, Colo. R. W. Beatty, NBS, Boulder, Colo.

28-30 American Ophthalmological Soc., Hot Springs, Va. 28- International Optical League Berlin Congress June 2 G. H. Giles, IOL, 65 Brook St., London, W.1, England

28- German Bunsen Society for Physical Chemistry June 3 61st General Assembly, Münster, Germany Carl Bosch Haus, Varrentrappstr. 40-42, Frankfurt am Main, Germany 29- $\begin{gathered}\text { World Meteorological Organization Congress, } \\ \text { Geneva, Switzerland }\end{gathered}$ 
31-

late

late

late

June

4-8

5-8

7-10

11-14

11-15

18-22

18-22

19-21

24-29

25-29

25-30

July

$8-14$

9-11

$9-20$

10-20

16-19

16-20

16-20

25-31

August
International Television Conference, London Institution of Electrical Engineers, Savoy Place, London, W.C.2, England

Interphoto Congress, Copenhagen International Photography and Cinematography Dealers Association, Steienvorstadt 63, Basel 10, Switzerland

Washington Symposium on Use of Artificial Satellites for Geodetic Purposes International Association of Geodes!. 18 mue Auber, Paris IX. France.

Journées de la Lumière, Grenoble W. Bing, Assoc. Francaise de la Lumière, 4 rue Ed.-Rousse, Paris 14 e, France

French Soc. of Physical Chemistry, Paris Secrétaire Général, Société de Chimie Physique, 10 rue Vauquelin, Paris 5e, France

24ème Congrès Annual du GAMS Secretariat, 1 rue $:$ G.

Boissier, Paris XV, France

Canadian Association of Physicists 17th Annuil Congress, Hamilton, Ontario,

Instrument Society of America, Seattle, Wash. Instrument-Automation Conference and Exhibit W. H. Kushnick, 3136th Ave., Pittsburgh 22, Pa.

Columbus Symposium on Molecular Structure and Spectroscopy R. A. Oetjen, Dept. Physics, Ohio State University, Columbus 10, Ohio

Eindhoven Congress on Magnetic and Electric Resonance and Relaxation D. J. Kroon, Natuurkundig Laboratorium, N.V. Philips' Gloelampenfabriek, Eindhoven, Netherlands

International Conference on Spectroscopy, Univ. of Maryland B. F. Scribner, National Bureau of Standards. Washinat'on 25, D.C.

APS Summer East Mtg., Evanston, Ill. K. $K$. Darrow, Columbia Univ., New York 27, N.Y.

ASTM National Meeting and Apparatus Exhibit, Statler Hotel, New York ASTM, 1916 Race St., Philadelphia 9, Pr.

Institute of Nuclear Magnetic Resonance, Buffalo H. A. Symanski, Chemistry, Canisius College, Buffalo 8, N.Y.

Copenhagen Symposium on Electromagnetic Theory and Antennas H. Lottrup Knudsen, Oster Voldgade $10 \mathrm{G}$, Copenhagen K. Denmark

Munich Symposium on Crystallographic Computing D. W. Smits, International Union of Crystallography, c/o Mathematisch Institut., Univ. of Groningen, Reitdiepskade 4, Groningen, Netherlands

6th International Commission on Glass, Washington, D.C. C. H. Hahner, General National Committee, c/o Glass Section, National Bureau of Standards, Washington D.C.

11th International Astrophysics Symposium, Liège $P$. Suings. Inst. of Astrophysics, Sciessin, Belgium

Summer Course in Optics Inst. of Optics, River Campus Station, Rochester $\mathbb{E} 0, N . Y$.

Summer Course on Techniques of Infrared Spectroscopy Director, Center for Continuation Study, Univ. of Minn., Minneapolis 14, Minn.

International Colloquium on Novae, Novoids and Supernovae, Lyons $J$. Dufay, U. de Lyons, rue de Cavenne, Lyons, France

IUPAP Conference on Paramagnetic Resonance, Jerusalem $W$. Low, Hebrew Univ., Jerusalem, Israel

International Conference on the Physics of Semiconductors, Exeter. IPPS, 47 Belgrave Square, London, S.W.1, England

Symposium in Commemoration of Fiftieth Anniversary of the Discovery of X-Ray Diffraction and of Crystal-structure Analysis, Munich $F$. Bopp, Inst. für Theoretische Physik, Sch:llingstr. 4-8, Munich, Germany

3rd International IUPAP Symposium on X-ray. Microscopy and X-ray Microanalysis, Stanford H. M. Pattee, Dept. Physics, Stanford Univ., Stanford, Calif.

2nd International Congress on Radiation Research, Harrogate A. Howard, Mount Vernon Hospital, Northwood, Middlesex, England
6-10 Society of Photographic and Instrumentation Engineers Meeting, Biltmore Hotel, New York.

6-17 MIT Infrared Summer Course J. M. Austin 7-103, MIT, Cambridge 39, Mass.

13-15 Interdisciplinary Conference on Electromagnetic Scattering, Potsdam, N.Y. M. Kerker, Clarkson College of Technology, Potsdam, N.Y.

14-16 Boulder International Conference on Precision Electromagnetic Measurements G. Birnbaum, Hughes Research Lab., Malibu, Calif.

14-18 Fisk Infrared Summer Course Director, Fisk Infrared Institute, Fisk Univ., Nashville, Tenn.

19-26 Sixth General Assembly of International Commission for Opties and Congress on Image Formation, Munich H. Schober, Institut für Medizinische Optik, Arnulstr. 205, Munich, Germany

21-24 International Symposium of Far Infrared Spectroscopy, Sheraton-Gibson Hotel, Cincinnati $F . F$. Bentley, ASD, Wright Patterson AF B, Ohio

21-28 Copenhagen International Congress on Acoustics F. H. B. Interslav, Tekniske Hojskole, Ostervoldgade 10, Copenhagen, Denmark

27-29 7th Symp. Ballistic Missile and Space Technology Aerospace Corp., P.O. Box 95085, Los Angeles 45, Calif.

27-29 APS Summer West Mtg., Seattle, Wash. H. A. Shugart, Physics, Univ. of Calif., Berkeley 4, Calif.

27-30 American Astronomical Society Meeting, Yale Univ. H. Smith, Yale U. Observatory, New Haven, Conn.

27-31 Infrared Gordon Conference, Meriden, N.H.

27- $\quad 2 n d$ International Conference on Information

Sept. 1 Processing, Munich IFIPS, 1634 Arch St.,

Sept. 1 Processing, Munich IFIPS, 1634 Arch St.,

30-Sept. 5 Symposium on Photo Interpretation, Delft Commission VII Intl. Training Centre for Aerial Survey, Kanaalweg 3, Delft, Netherlands

September

Manchester G. Allen, Burlington House, London, W. 1, England

3-7 Brussels International Symposium on Information Theory F. L. Stumpers, Philips Research Labs, Eindhoven, Netherlands

5-7 Emittance and Reflection of Radiation from Various Materials and Space Applications Univ. of Dayton

7-12 Kycto International Conference on Crystal Lattice Defects R. R. Hasiguti, Univ. Tokyo, Bunkyoku, Tokyo, Japan

9-14 Dallas Technical Conference of the Illuminating Engineering Society

10-15 IUPAC Tokyo International Symposium on Molecular Structure and Spectroscopy Science Council of Japan, Ueno Park, Tolyo, Japan

16-20 Electrochemical Society Meeting, Statler Hilton Hotel, Boston, Mass.

17-19 9tl Ottawa Symposium on Applied Spectroscopy B. Farrar, J. T. Donald \& Co. Ltd., 1181 Guy St., Montreal 25, Que., Canada.

17-22 6th Intl. Congress on High Speed Photography, Scheveningen J. G. A. de Graaf, 14, Burgemeester de Monchyplein, The Hague, Netherlands

24-28 Union of German Physical Societies, Stuttgart H. Franke, Verband Deutscher Physik. Gesell., Gänseheide 15A, Stuttgart, Germany

October

Infrared Information Symposium Fall Meeting Eastern States

URSI Montreal Meeting $M$. G. George, Thayer School of Engineering, Dartmouth College, Hanover, N.H.

Varian Annual NMR-EPR Workshop Varian Assoc., Hansen Way, Palo Alto, Calif.

4-6 Optical Society of America 47th Annual Meeting, Manger Hotel, Rochester Hilda Kingslake, 56 Westland Ave., Rochester, N.Y.

5 OSA Annual Business Meeting, Manger Hotel, Rochester, N.Y.

15-19 Instrument Society of America 17th Annual Instrument-Automation Conference and Exhibit $W . H$. Kushnick, 3196th Ave., Pittsburgh 22, Pa. 